\title{
VARIABILIDADE ESPACIAL DA TEXTURA DE UM LATOSSOLO VERMELHO EUTROFÉRRICO SOB CULTIVO DE CANA-DE-AÇÚCAR ${ }^{1}$
}

\author{
ZIGOMAR M. SOUZA ${ }^{2}$, JOSÉ MARQUES JÚNIOR ${ }^{3}$, GENER T. PEREIRA ${ }^{4}$, \\ DIOGO M. BARBIERI ${ }^{5}$
}

RESUMO: Estudos regionais mais detalhados, utilizando modelos de paisagem e geoestatística, têm demonstrado que, em áreas consideradas homogêneas, sob uma única classe de solo, existe dependência espacial de atributos granulométricos. Visando a avaliar a variabilidade espacial de atributos granulométricos em Latossolo Vermelho eutroférrico, foram feitas amostragens do solo em intervalos regulares de $50 \mathrm{~m}$, em forma de malha, totalizando 306 pontos de amostragem. Foram coletadas amostras nas profundidades de 0-0,2 m e 0,6-0,8 m para a determinação da argila, silte, areia total (AT), areia grossa (AG), areia média (AM), areia fina (AF) e areia muito fina (AMF). Os dados foram submetidos à análise estatística descritiva, geoestatística e interpolação por krigagem. Os valores do coeficiente de variação apresentaram-se baixos para argila, médios para silte, AT, AF, AM e AMF e altos para AG. Observou-se ocorrência de dependência espacial para todas as variáveis com grau moderado de dependência espacial, com os maiores alcances ocorrendo na profundidade de 0-0,2 m. Os latossolos, apesar de serem homogêneos, mesmo em áreas de mesma classe de solo e manejo, apresentaram variabilidade diferenciada para os atributos granulométricos.

PALAVRAS-CHAVE: geoestatística, krigagem, atributos físicos.

\section{SPATIAL VARIABILITY OF THE TEXTURE IN AN EUTRUDOX RED LATOSOL UNDER SUGARCANE CROP}

SUMMARY: Regional studies where geostatistic is applied for describing landscape models have shown that, even in places previously considered homogenous, different spatial dependencies have been found and these had been related to crop productivity. In order to analyze the physical properties spatial variability models of an eutrudox Red Latosol, a regular grid containing 306 points spaced at separation distances of 50 meters was installed in southern Brazil. Soil samples were collected at 0-0.2 $\mathrm{m}$ and $0.6-0.8 \mathrm{~m}$ in order to determine clay, silt, sand total, sand thick, sand medium, sand fine and sand very fine contents. Data were submitted to descriptive statistics, geostatistic and in sequence to kriging analyzes. The coefficient of variation of studied properties was low for clay and, medium for silt, sand total, sand medium, sand fine and sand very fine and, high for sand thick. All the spatial variability models had moderate variability dependence, with higher ranges for $0-0.2 \mathrm{~m}$ of depth. The results showed that the considered homogeneous characteristics of oxisols submitted to the same soil management is not so evident and spatial variability of texture attributes can be distinguished from each other.

KEYWORDS: geostatistics, kriging, physical attributes.

\footnotetext{
${ }^{1}$ Parte da Tese de Doutorado do primeiro autor, financiada pela FAPESP.

${ }^{2}$ Doutorando do Departamento de Solos e Adubos, Faculdade de Ciências Agrárias e Veterinárias - UNESP, Via de Acesso Prof. Paulo Donato Castellane, km 5, Jaboticabal - SP, Bolsista da FAPESP, e-mail:zigomar@ fcav.unesp.br

${ }^{3}$ Prof. Dr., Departamento de Solos e Adubos, FCAV/UNESP, Jaboticabal - SP.

${ }^{4}$ Prof. Dr., Departamento de Ciências Exatas, FCAV/UNESP, Jaboticabal - SP.

${ }^{5}$ Aluno de Iniciação Científica, FCAV/UNESP, Jaboticabal - SP.

Recebido pelo Conselho Editorial em: 11-3-2003

Aprovado pelo Conselho Editorial em: 11-3-2004
} 


\section{INTRODUÇÃO}

No Brasil, são poucos os estudos que dimensionam a variabilidade espacial de atributos físicos em uma unidade de mapeamento de solo. Os poucos trabalhos brasileiros existentes nessa área têm utilizado escalas espaciais que representam pequenas distâncias no terreno. $\mathrm{O}$ conhecimento da distribuição das propriedades do solo no campo é importante para o refinamento das práticas de manejo e avaliação dos efeitos da agricultura sobre a qualidade ambiental (CAMBARDELLA et al., 1994).

No nordeste do Estado de São Paulo, empresas agrícolas mapearam grandes áreas de seus solos, em diversos níveis de detalhe, para o entendimento da necessidade de manejo mais adequado das culturas, notadamente a cana-de-açúcar. Em áreas de solos desenvolvidos de basalto, os mapas apresentam, via de regra, uma única cor, indicando baixa variabilidade da ocorrência de classe de solos e, conseqüentemente, homogeneidade dos seus atributos físicos. Nesse caso, poucas alterações nas práticas de manejo são recomendadas para essas áreas. Entretanto, estudos regionais mais detalhados, utilizando modelos de paisagem e geoestatística (MARQUES JÚNIOR \& LEPSCH, 2000; POCAY, 2000 e SOUZA, 2001), têm demonstrado que, em áreas consideradas homogêneas, sob uma única classe de solo, existe dependência espacial de atributos granulométricos a curtas distâncias em grau suficiente para interferir na produtividade das culturas. HALL \& OLSON (1991) mencionam a importância da classificação dos solos como auxiliar ao manejo das culturas, porém ressaltam que o registro das relações do solo com a paisagem como causa das variações de propriedades tem sido ignorado. Nesse sentido, os mapeamentos da variabilidade espacial de atributos granulométricos nessas áreas representam um esforço importante para o manejo de aplicação de insumos com taxas variadas.

A análise de dados obtidos em condições de campo tem apresentado dificuldade nas diversas áreas da Ciência, devido à variabilidade espacial. Quando se trata da variabilidade do solo, as dificuldades tornam-se maiores porque, sendo a formação do solo resultante de uma série de fatores, $o$ produto final é bastante heterogêneo em relação às suas características e propriedades (SILVA et al., 1989). Em áreas cultivadas, além da variabilidade natural do solo, existem fontes adicionais de variabilidade devidas ao manejo exercido pelo homem, uma das quais é o cultivo em linhas e a conseqüente aplicação localizada de fertilizantes (JOHNSON et al., 1996 e SOUZA et al., 1997).

$\mathrm{O}$ objetivo deste trabalho foi caracterizar a variabilidade espacial de atributos granulométricos em um Latossolo Vermelho eutroférrico, sob cultivo de cana-de-açúcar, associados a compartimentos da paisagem.

\section{MATERIAL E MÉTODOS}

A área de estudo localiza-se no nordeste do Estado de São Paulo, no município de Guariba - SP. As coordenadas geográficas são $21^{\circ} 19^{\prime}$ de latitude sul e $48^{\circ} 13^{\prime}$ de longitude oeste, com altitude média de $640 \mathrm{~m}$. O clima da região, segundo a classificação de Köeppen, é do tipo mesotérmico com inverno seco (Cwa), com precipitação média de $1.400 \mathrm{~mm}$ e chuvas concentradas no período de novembro a fevereiro. A vegetação natural era constituída por floresta tropical subcaducifólia e mata ciliar.

O relevo é predominantemente suave ondulado com declividades médias variando de 3 a $8 \%$. A área experimental vem sendo cultivada há mais de 30 anos com cana-de-açúcar. O solo da área foi classificado como Latossolo Vermelho eutroférrico, textura muito argilosa (LVef) (EMBRAPA, 1999).

No terço inferior da encosta, por meio da análise detalhada das formas do relevo e da variação do gradiente do declive, caracterizaram-se dois compartimentos (I e II, Figura 1), sob os quais os solos foram amostrados nos pontos de cruzamento de uma malha, com intervalos regulares de $50 \mathrm{~m}$, 
perfazendo um total de 206 pontos nas profundidades de 0-0,2 m e 0,6-0,8 m. Acrescentaram-se mais quatro submalhas com 25 pontos (refinamento), com intervalos regulares de $10 \mathrm{~m}$ (100 pontos), totalizando-se 306 pontos de amostragem em uma área de 42 ha. A composição granulométrica foi determinada pelo método da pipeta, utilizando solução de $\mathrm{NaOH} 0,1 \mathrm{~N}$ como dispersante químico e agitação mecânica em aparato de baixa rotação por $16 \mathrm{~h}$, seguindo metodologia proposta pela EMBRAPA (1997), com modificações. Utilizaram-se $15 \mathrm{~g}$ de areia lavada com diâmetro entre 0,5 1,0 mm para auxiliar na dispersão física das partículas. A fração argila foi separada por sedimentação, de acordo com a lei de Stokes, sendo a fração silte determinada por diferença. A fração areia, após secagem e pesagem, foi subdividida em cinco subfrações: areia muito grossa (AMG), areia grossa $(A G)$, areia média $(A M)$, areia fina $(A F)$ e areia muito fina (AMF), em peneiras de $2-1 \mathrm{~mm} ; 1-0,5 \mathrm{~mm}$; 0,5-0,25 mm;0,25-0,10 $\mathrm{mm}$ e 0,10-0,05 $\mathrm{mm}$, respectivamente.

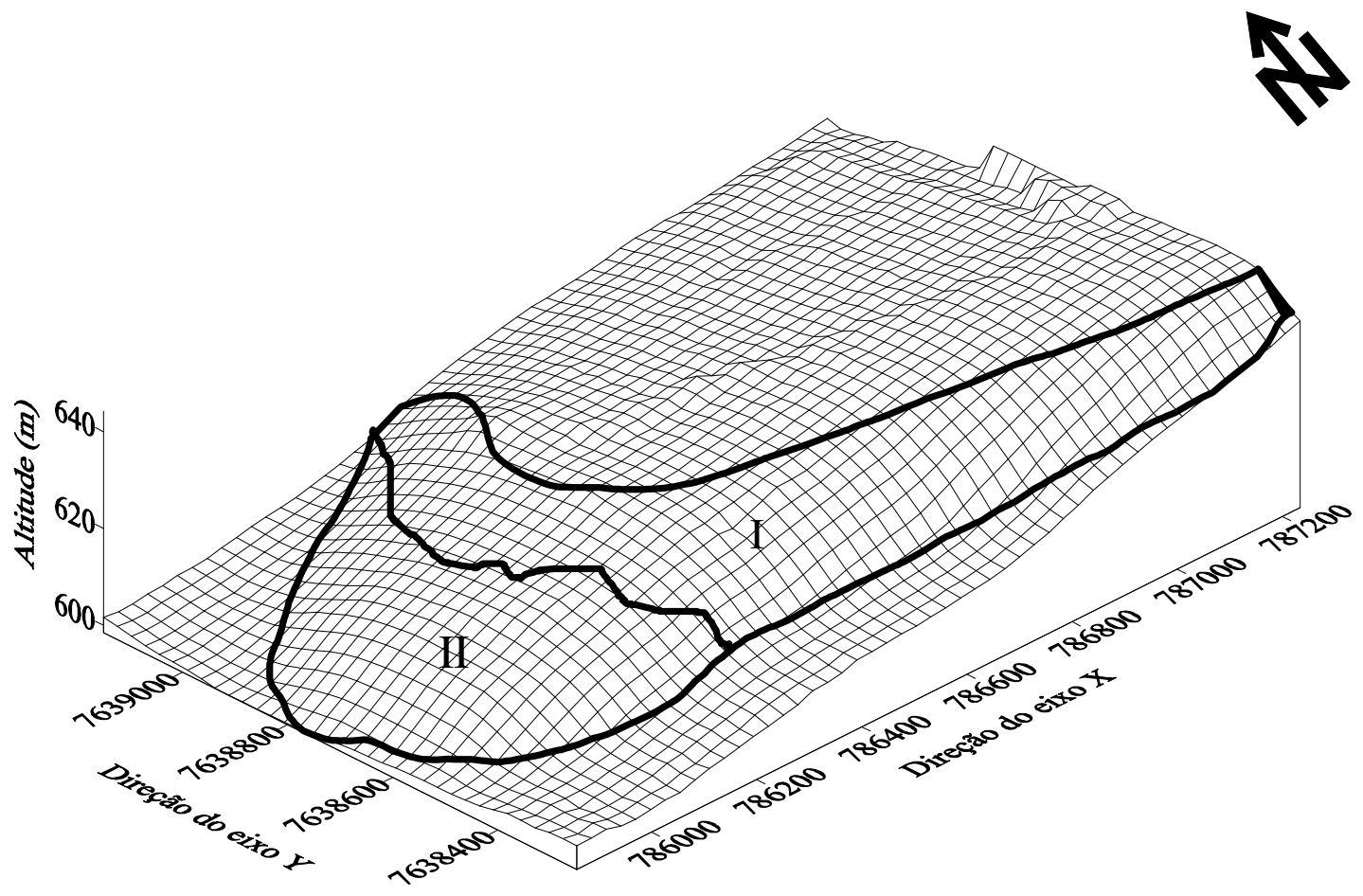

FIGURA 1. Mapa da área de estudo, com compartimentos identificados (I e II).

Os atributos granulométricos do solo foram analisados por meio da análise estatística descritiva e de técnicas geoestatísticas. A hipótese de normalidade dos dados foi testada pelo teste de KolmogorovSmirnov, SAS (1995). A dependência espacial foi analisada por meio de ajuste de semivariograma (VIEIRA et al., 1983 e ROBERTSON, 1998), com base nas pressuposições de estacionariedade da hipótese intrínseca, estimado por:

$$
\hat{\gamma}(\mathrm{h})=\frac{1}{2 \mathrm{~N}(\mathrm{H})} \sum_{\mathrm{i}=1}^{\mathrm{N}(\mathrm{h})}\left[\mathrm{Z}\left(\mathrm{x}_{\mathrm{i}}\right)-\mathrm{Z}\left(\mathrm{x}_{\mathrm{i}}+\mathrm{h}\right]^{2}\right.
$$

em que, $\mathrm{N}$ (h) é o número de pares experimentais de observações $\mathrm{Z}\left(\mathrm{x}_{\mathrm{i}}\right)$ e $\mathrm{Z}\left(\mathrm{x}_{\mathrm{i}}+\mathrm{h}\right)$ separados por uma distância h. O semivariograma é representado pelo gráfico $\hat{\gamma}(h)$ versus h. Do ajuste de um modelo matemático aos valores calculados de $\hat{\gamma}(h)$, são estimados os coeficientes do modelo teórico para o semivariograma (o efeito pepita, $\mathrm{C}_{0}$; variância estrutural, $\mathrm{C}_{1}$; patamar, $\mathrm{C}_{0}+\mathrm{C}_{1}$, e o alcance, a). Os modelos de semivariogramas considerados foram o esférico, o exponencial e o gaussiano 
(McBRATNEY \& WEBSTER, 1986). A razão entre a variância estrutural e o patamar $\mathrm{C}_{1} /\left(\mathrm{C}_{1}+\mathrm{C}_{0}\right)$, expressa em porcentagem, permitiu a classificação do grau de dependência espacial, a qual, segundo ROBERTSON (1998), é considerada fraca se essa razão for $\leq 25 \%$, moderada quando essa razão estiver entre 25 e $75 \%$, e forte se a razão for $>75 \%$.

Esses modelos foram ajustados por meio do programa $\mathrm{GS}^{+}$(ROBERTSON, 1998). Em caso de dúvida entre mais de um modelo para o mesmo semivariograma, considerou-se o melhor $\mathrm{R}^{2}$ (coeficiente de determinação) e a menor SQR (soma de quadrados do resíduo). Para a elaboração dos mapas de distribuição espacial das variáveis, foi utilizado o programa Surfer (GOLDEN SOFTWARE, 1999).

\section{RESULTADOS E DISCUSSÃO}

Os valores da mediana e média das frações granulométricas analisadas foram similares (Tabela 1), indicando distribuições simétricas, o que pode ser confirmado pelos valores de assimetria próximos de zero, com exceção da areia grossa (AG), em ambas as profundidades, e areia fina, na profundidade de $0,6-0,8 \mathrm{~m}$.

A variável argila apresentou distribuição normal nas duas profundidades estudadas e silte na profundidade de 0,6-0,8 m; as demais variáveis estudadas não apresentaram normalidade. VIEIRA (1997), ARAUJO (2002) e GONÇALVES \& FOLEGATTI (2002) encontraram normalidade para a variável argila, trabalhando com amostragem em malha regular. Resultados semelhantes para a variável silte também foram observados por VIEIRA (1997), GONÇALVES et al. (2001) e SOUZA et al. (2003). A não-normalidade para as areias também foi verificada por POCAY (2000), ARAUJO (2002), EGUCHI et al. (2002) e SOUZA et al. (2003).

Como salientado por WARRICK \& NIELSEN (1980) e WEBSTER (1985), em se tratando de dados obtidos na natureza, o ajuste a uma distribuição teórica é apenas aproximado. De acordo com CRESSIE (1991), a normalidade dos dados não é uma exigência da geoestatística, sendo conveniente apenas que a distribuição não apresente caudas muito alongadas, o que pode comprometer os resultados. Assim, a partir dos resultados da análise exploratória dos dados, é possível admitir que essas variáveis apresentam distribuições suficientemente simétricas para o estudo geoestatístico.

Os valores do coeficiente de variação (C.V.) referentes à granulometria indicaram variabilidade baixa para argila $(4,77$ - 3,05\%); resultados semelhantes também foram encontrados por COELHO FILHO et al. (2001) e GONÇALVES et al. (2001); média para silte (16,64 - 12,71\%), AT (14,98 $12,25 \%), \operatorname{AM}(22,09-16,22 \%), \operatorname{AF}(14,92-10,52 \%)$ e AMF $(21,23-19,78 \%)$ e alta para a AG $(40,58$ - 35,12\%), concordando com os resultados obtidos por POCAY (2000) e SOUZA (2001), nas profundidades de 0-0,2 m e 0,6-0,8 m, respectivamente. O coeficiente de variação foi menor na camada de 0-0,2 m em relação à camada de 0,6-0,8 m para todas as variáveis estudadas. Os resultados da Tabela 1 revelaram que o solo é de textura muito argilosa quando avaliado pela média e valores de mínimo e máximo.

Argila e areia dos latossolos são atributos estáveis, ou seja, modificam-se pouco ao longo do tempo por ação do intemperismo, portanto apresentam baixos valores de C.V. No presente estudo, o atributo areia apresentou maiores valores de C.V., provavelmente indicando a influência da posição da paisagem, devido a processos erosionais ou deposicionais, associados ao fluxo de água, concordando com os estudos de POCAY (2000) e SOUZA (2001).

Para as variáveis argila, silte, areia grossa, areia fina e areia muito fina, na profundidade de 0-0,2 m e com exceção da argila na profundidade 0,6-0,8 m, estimaram-se os semivariogramas para os resíduos da tendência linear. A remoção dessa tendência foi necessária porque o semivariograma para os dados originais apresentou crescimento sem limites com a distância. 
TABELA 1. Estatística descritiva para as variáveis argila, silte, AT, AG, AM, AF e AMF, em g kg-1, de amostras coletadas na malha, em duas profundidades do solo.

\begin{tabular}{|c|c|c|c|c|c|c|c|}
\hline \multirow{2}{*}{ Parâmetro } & \multicolumn{7}{|c|}{ Variáveis Granulométricas } \\
\hline & Argila & Silte & $\mathrm{AT}^{1}$ & $\mathrm{AG}^{2}$ & $\mathrm{AM}^{3}$ & $\mathrm{AF}^{4}$ & $\mathrm{AMF}^{5}$ \\
\hline \multicolumn{8}{|c|}{ Profundidade $0-0,2 \mathrm{~m}$} \\
\hline Média & 631,69 & 183,23 & 177,25 & 15,09 & 53,39 & 68,88 & 43,07 \\
\hline Mediana & 630,50 & 181,54 & 175,11 & 11,12 & 51,17 & 70,02 & 41,73 \\
\hline Mínimo & 590 & 120 & 130 & 10 & 30 & 50 & 30 \\
\hline Máximo & 700 & 260 & 240 & 30 & 80 & 90 & 60 \\
\hline Variância & 708,57 & 536,71 & 451,15 & 47,36 & 129,23 & 105,64 & 83,64 \\
\hline Assimetria & 0,21 & 0,25 & 0,23 & 1,29 & 0,46 & 0,38 & 0,18 \\
\hline Curtose & $-0,46$ & 1,13 & 1,47 & $-0,26$ & 0,12 & $-0,48$ & $-0,78$ \\
\hline C.V. $(\%)$ & 4,77 & 16,64 & 14,98 & 40,58 & 22,09 & 14,92 & 21,23 \\
\hline $\mathrm{p}^{6}$ & $0,08^{*}$ & 0,15 & 0,16 & 0,21 & 0,18 & 0,14 & 0,20 \\
\hline \multicolumn{8}{|c|}{ Profundidade $0,6-0,8 \mathrm{~m}$} \\
\hline Média & 705,02 & 149,96 & 142,90 & 12,38 & 43,49 & 54,90 & 36,66 \\
\hline Mediana & 703,11 & 150,01 & 140,50 & 9,11 & 41,22 & 52,06 & 38,19 \\
\hline Mínimo & 660 & 100 & 100 & 10 & 20 & 30 & 20 \\
\hline Máximo & 760 & 200 & 190 & 30 & 80 & 80 & 60 \\
\hline Variância & 465,66 & 228,52 & 306,59 & 28,06 & 150,68 & 114,90 & 97,70 \\
\hline Assimetria & 0,45 & $-0,29$ & 0,73 & 2,16 & 1,24 & 0,88 & 0,50 \\
\hline Curtose & $-0,20$ & $-0,65$ & 0,26 & 2,78 & 1,43 & 0,36 & $-0,12$ \\
\hline C.V. $(\%)$ & 3,05 & 12,71 & 12,25 & 35,12 & 16,22 & 10,52 & 19,78 \\
\hline $\mathrm{p}$ & $0,07^{*}$ & $0,08^{*}$ & 0,09 & 0,22 & 0,22 & 0,23 & 0,19 \\
\hline
\end{tabular}

1 - areia total; 2 - areia grossa; 3 - areia média; 4 - areia fina; 5 - areia muito fina; 6 - estatística do teste KolmogorovSmirnov; * significativo a de $5 \%$.

Os resultados da análise geoestatística (Tabela 2 e Figura 2) mostraram que todas as variáveis analisadas apresentaram dependência espacial nas duas profundidades estudadas. Na profundidade 00,2 m, os dados das variáveis argila, silte, AG e AM ajustaram-se ao modelo esférico, e as variáveis AT, AF e AMF ajustaram-se ao modelo exponencial, com $\mathrm{R}^{2}$ acima de 0,94 . Na profundidade 0,60,8 m, todas as variáveis foram ajustadas ao modelo exponencial, com exceção da argila e AF que se ajustaram ao modelo esférico, com $\mathrm{R}^{2}$ acima de 0,90 . $\mathrm{O}$ modelo que se ajustou com maior freqüência aos dados granulométricos foi o exponencial, juntamente com o esférico, sendo que BERTOLANI et al. (2000) afirmam que são esses os modelos que mais se ajustam aos dados de atributos físicos do solo.

A análise da relação $\mathrm{C}_{1} /\left(\mathrm{C}_{0}+\mathrm{C}_{1}\right)$ dos atributos granulométricos do solo (Tabela 2) mostrou que as variáveis argila, silte e AMF apresentaram dependência espacial moderada, e as variáveis AT, AG, AM e AF apresentaram dependência espacial forte na profundidade de 0-0,2 m; na profundidade de 0,6-0,8 m, todas as variáveis em estudo tiveram dependência espacial moderada, segundo a classificação proposta por ROBERTSON (1998). As variáveis apresentaram diferentes alcances, sendo que, na camada superficial, as variáveis AG e AM apresentaram os maiores alcances, e silte e AF os menores $(366,8 ; 287,7 ; 157,0$ e $199,6 \mathrm{~m}$, respectivamente). Na profundidade de $0,6-0,8 \mathrm{~m}$, os alcances foram menores em relação à profundidade de 0-0,2 m, com máximo de 215,2 m para a variável argila e mínimo de 57,9 m para variável AT, indicando a maior descontinuidade na distribuição espacial das propriedades do solo na camada mais profunda. Isso está de acordo com um importante conceito em gênese do solo, que o maior tempo relativo de exposição dos horizontes superficiais condiciona sua maior taxa de intemperismo (BUOL et al., 1997). Esse fato pode ter contribuído para aumentar o 
alcance da dependência espacial, caracterizando maior continuidade na distribuição das variáveis na camada mais superficial da área estudada, concordando com estudo de SOUZA (2001) e ARAUJO (2002), trabalhando com malhas regulares espaçadas em $50 \mathrm{~m}$.

TABELA 2. Modelos e parâmetros estimados dos semivariogramas experimentais para as variáveis argila, silte, areia total, areia grossa, areia média, areia fina e areia muito fina $\left(\mathrm{g} \mathrm{kg}^{-1}\right) \mathrm{de}$ amostras coletadas na malha, em duas profundidades do solo.

\begin{tabular}{|c|c|c|c|c|c|c|c|}
\hline \multirow[b]{2}{*}{$\begin{array}{l}\text { Parâmetros dos } \\
\text { Semivariogramas }\end{array}$} & \multicolumn{7}{|c|}{ Atributos Granulométricos } \\
\hline & Argila & Silte & $\begin{array}{c}\text { Areia } \\
\text { total }\end{array}$ & $\begin{array}{l}\text { Areia } \\
\text { grossa }\end{array}$ & $\begin{array}{l}\text { Areia } \\
\text { média }\end{array}$ & $\begin{array}{l}\text { Areia } \\
\text { fina }\end{array}$ & $\begin{array}{c}\text { Areia } \\
\text { muito fina }\end{array}$ \\
\hline \multicolumn{8}{|c|}{ Profundidade de $0-0,2 \mathrm{~m}$} \\
\hline Modelo & ${ }^{1}$ Esf & Esf & ${ }^{2} \operatorname{Exp}$ & Esf & Esf & Exp & $\operatorname{Exp}$ \\
\hline Efeito pepita $\left(\mathrm{C}_{0}\right)$ & 197,0 & 336,0 & 123,0 & 8,9 & 26,2 & 28,3 & 51,0 \\
\hline Patamar $\left(\mathrm{C}_{0}+\mathrm{C}_{1}\right)$ & 427,2 & 387,0 & 657,8 & 45,6 & 109,5 & 164,0 & 65,1 \\
\hline Alcance (a) & 230,1 & 157,7 & 210,0 & 366,8 & 287,7 & 199,6 & 204,0 \\
\hline${ }^{3} \mathrm{C}_{1} /\left(\mathrm{C}_{1}+\mathrm{C}_{0}\right)$ & 68 & 53 & 84 & 83 & 81 & 85 & 56 \\
\hline${ }^{4} \mathrm{R}^{2}$ & 0,99 & 0,94 & 0,97 & 0,98 & 0,98 & 0,98 & 0,97 \\
\hline${ }^{5} \mathrm{SQR}$ & 845 & 42,5 & 82,8 & 39,8 & 246 & 261 & 71,4 \\
\hline \multicolumn{8}{|c|}{ Profundidade de $0,6-0,8 \mathrm{~m}$} \\
\hline Modelo & Esf & $\operatorname{Exp}$ & $\operatorname{Exp}$ & $\operatorname{Exp}$ & $\operatorname{Exp}$ & Esf & $\operatorname{Exp}$ \\
\hline Efeito pepita $\left(\mathrm{C}_{0}\right)$ & 152,9 & 105,0 & 83,6 & 3,5 & 17,3 & 21,2 & 42,2 \\
\hline Patamar $\left(\mathrm{C}_{0}+\mathrm{C}_{1}\right)$ & 263,9 & 105,1 & 189,5 & 10,7 & 23,5 & 29,6 & 46,7 \\
\hline Alcance (a) & 215,2 & 83,1 & 57,9 & 209,8 & 126,3 & 180,3 & 88,7 \\
\hline $\mathrm{C}_{1} /\left(\mathrm{C}_{1}+\mathrm{C}_{0}\right)$ & 63 & 50 & 69 & 75 & 57 & 58 & 52 \\
\hline $\mathrm{R}^{2}$ & 0,98 & 0,98 & 0,98 & 0,90 & 0,99 & 0,95 & 0,94 \\
\hline SQR & 1438 & 114 & 238 & 6,4 & 1,4 & 34,7 & 70,3 \\
\hline
\end{tabular}

1 - esférico; 2 - exponencial; 3 - grau de dependência espacial; 4 - coeficiente de determinação; 5 - soma dos quadrados do resíduo.

Os parâmetros dos modelos de semivariogramas ajustados (Figura 2) foram utilizados para estimar valores em locais não amostrados por meio da krigagem (Figura 3). Os mapas espaciais das variáveis argila e areia total têm comportamento inverso, quando comparados entre si. O maior teor da variável argila está no compartimento I, relativamente menos declivoso, em relação ao compartimento II. A variável areia total apresenta o mesmo comportamento das variáveis AG, AM e AF com maiores teores no compartimento de maior declive da área, influenciado pelas maiores taxas de erosão nessa posição da paisagem (SOUZA et al., 2002).

Essa informação sugere retenção de água e teores de íons diferenciados para esses compartimentos, podendo interferir no comportamento da cultura. FRAISSE et al. (1999) e FRIDGES et al. (2000) afirmam que a utilização da altitude, declividade e formas da paisagem do solo para delinear zonas de manejo para atributos granulométricos e químicos, em diferentes tipos de solos, são de extrema importância. MACHADO (1994) afirma que a posição topográfica dos solos na paisagem, influindo na drenagem do solo, é um dos principais fatores condicionantes da variabilidade espacial dos solos.

A amplitude das variáveis estudadas enfoca que, numa mesma classe de solos, conceitualmente considerada altamente intemperizada (Latossolo Vermelho eutroférrico), se observa que ocorre variabilidade espacial dos atributos granulométricos. Considerando que os teores de argila estão relacionados a compartimentos com declive diferenciado e que esse atributo é estável no solo, sugerese que essas pequenas variações do gradiente do declive possam contribuir para a identificação de limites de precisão entre locais específicos da paisagem. 


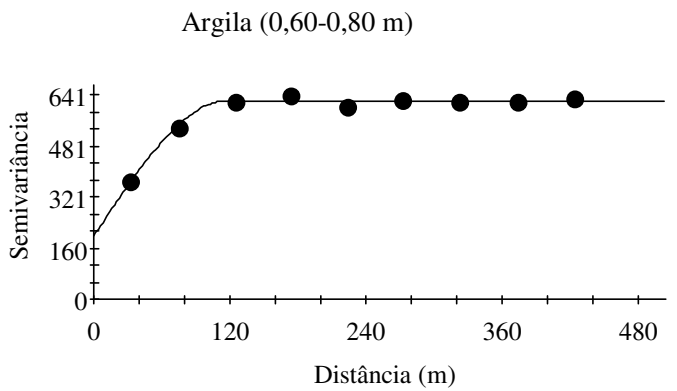

Esf $(197 ; 427,20 ; 230,1)$

Silte $(0,00-0,20 \mathrm{~m})$

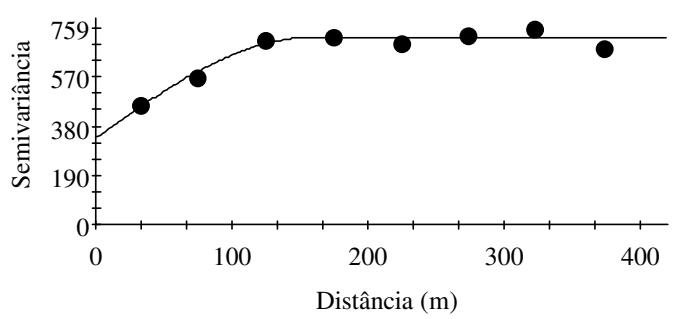

Esf $(336 ; 387 ; 157,70)$

AT $(0,00-0,20 \mathrm{~m})$

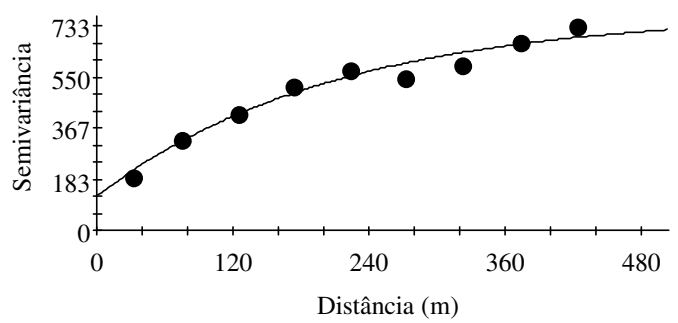

$\operatorname{Exp}(123 ; 657,80 ; 210)$

$\operatorname{AM}(0,00-0,20 \mathrm{~m})$

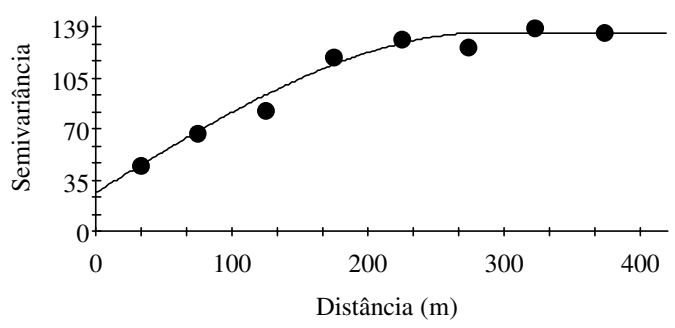

Esf $(26,20 ; 109,50 ; 287,70)$

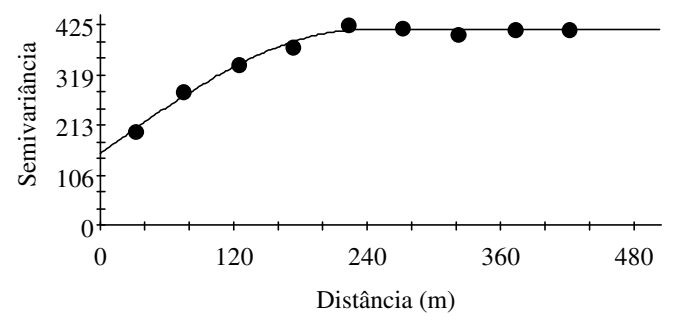

Esf (152,9; 263,9; 215,2)

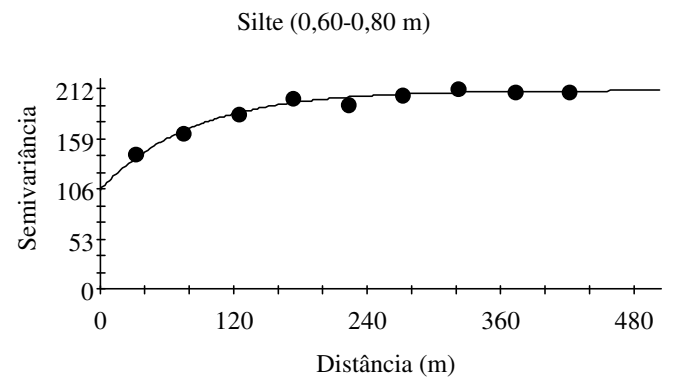

$\operatorname{Exp}(105 ; 105,10 ; 83,10)$

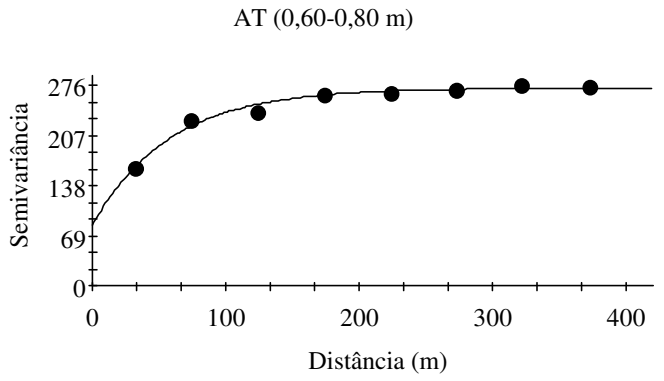

$\operatorname{Exp}(83,60 ; 189,50 ; 57,90)$

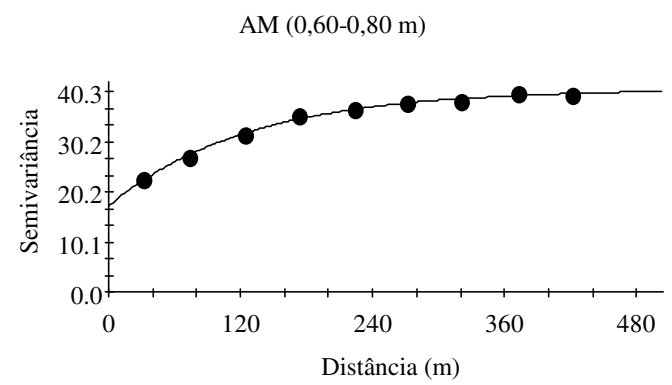

$\operatorname{Exp}(17,32 ; 23,53 ; 126,30)$

FIGURA 2. Semivariogramas das variáveis granulométricas argila, silte, areia total e areia média $\left(\mathrm{g} \mathrm{kg}^{-1}\right)$ nas profundidades de 0-0,2 m e 0,6-0,8 m. Esf e Exp $\left(\mathrm{C}_{0} ; \mathrm{C}_{0}+\mathrm{C}_{1} ;\right.$ a) são o modelo esférico e exponencial ajustado, $\mathrm{C}_{\mathrm{o}}=$ efeito pepita; $\mathrm{C}_{0}+\mathrm{C}_{1}=$ patamar; $\mathrm{a}=$ alcance. 

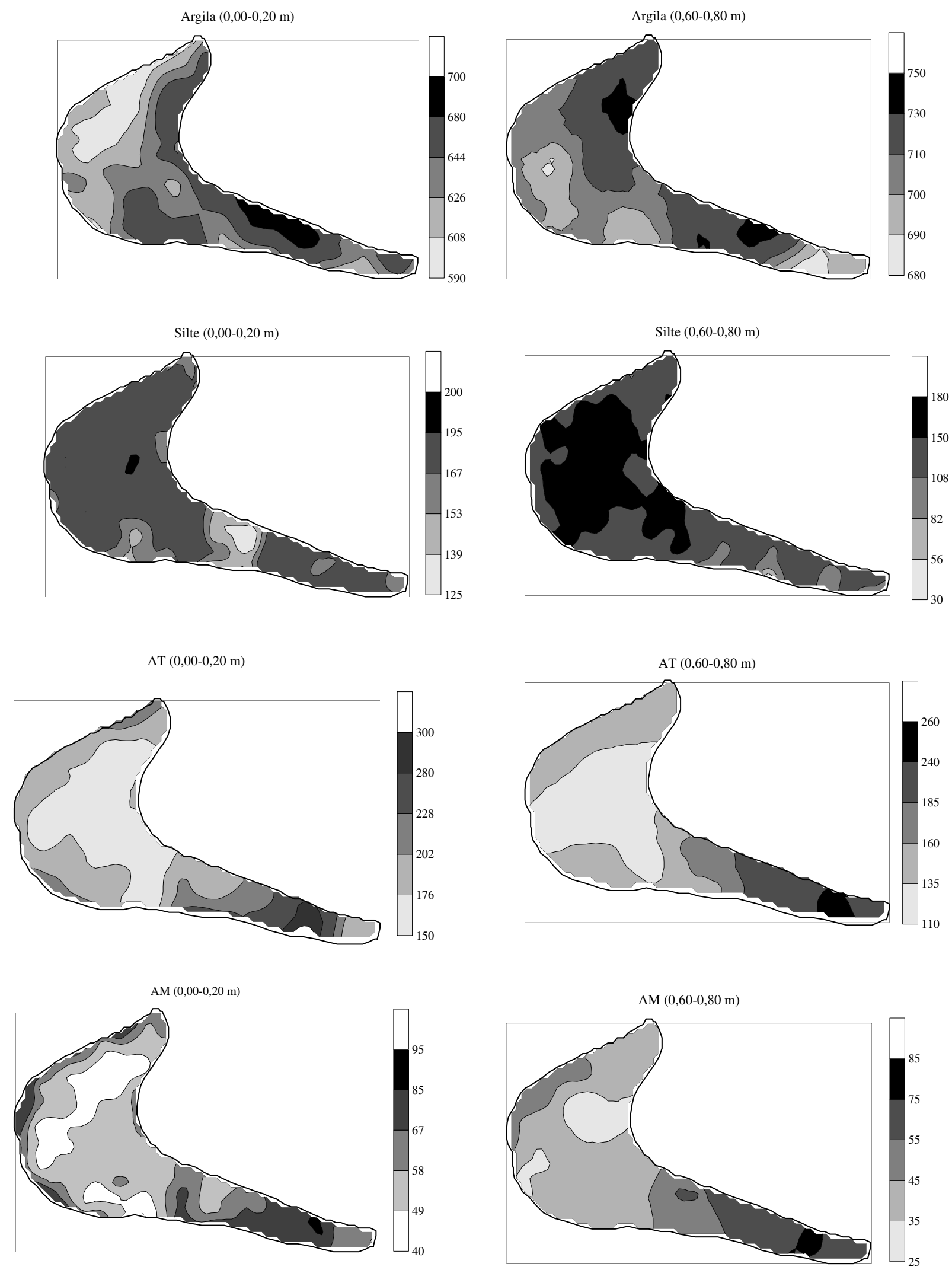

FIGURA 3. Distribuição espacial das variáveis argila $\left(\mathrm{g} \mathrm{kg}^{-1}\right)$, silte $\left(\mathrm{g} \mathrm{kg}^{-1}\right)$, areia total $\left(\mathrm{g} \mathrm{kg}^{-1}\right)$, areia média $\left(\mathrm{g} \mathrm{kg}^{-1}\right)$, nas profundidades de $0-0,2 \mathrm{~m} \mathrm{e} 0,6-0,8 \mathrm{~m}$, na área estudada. 


\section{CONCLUSÕES}

A variabilidade do solo medida pelo coeficiente de variação registrou-se baixa para argila, média para silte, areia total, areia média, areia fina e areia muito fina, e alta para areia grossa, nas profundidades estudadas.

As variáveis estudadas apresentaram estrutura de dependência espacial. Os maiores alcances foram observados na profundidade de 0-0,2 m para todos os atributos estudados, refletindo os efeitos do maior tempo de exposição dos horizontes superficiais nas maiores taxas relativas de intemperismo.

Os latossolos sob cultivo de cana-de-açúcar, apesar de serem considerados homogêneos, apresentam variabilidade diferenciada para os atributos granulométricos.

A identificação de compartimentos da paisagem na área de estudo mostrou-se muito eficiente na compreensão da variabilidade espacial dos atributos estudados.

\section{REFERÊNCIAS BIBLIOGRÁFICAS}

ARAUJO, A.A.V. Variabilidade espacial de propriedades químicas e granulométricas do solo na definição de zonas homogêneas de manejo. 2002. 80 f. Dissertação (Mestrado em Ciência do Solo) Faculdade de Ciências Agrárias e Veterinárias, Universidade Estadual de São Paulo, Jaboticabal.

BERTOLANI, F.C.; GONZÁLEZ, A.P.; LIÑARES, M.L.; VÁZQUEZ, E.V.; MIRANDA, J.G.V.M. Variabilidade espacial da rugosidade superficial do solo medida com rugosímetros de agulhas e laser. Bragantia, Campinas, v.59, n.2, p.227-34, 2000.

BUOL, S.W.; HOLE, F.D.; MCCRACKEN, R.J.; SOUTHARD, R.J. Soil genesis and classification. Iowa: Iowa State University Press, 1997. 527 p.

CAMBARDELLA, C.A.; MOORMAN, T.B.; NOVAK, J.M.; PARKIN, T.B.; KARLEN, D.L.; TURCO, R.F.; KONOPKA, A.E. Field-scale variability of soil properties in Central Iowa Soils. Soil Science Society of American Journal, Madison, v.58, n.5, p.1501-11, 1994.

COELHO FILHO, M.A.; COELHO, R.D.; GONÇALVES, A.C.A. Variabilidade espacial de variáveis físico-hídricas do solo em um pomar de lima ácida Tahiti, irrigado por microaspersão. Revista Brasileira de Engenharia Agrícola e Ambiental, Campina Grande, v.5, n.2, p.239-46, 2001.

CRESSIE, N. Statistics for spatial data. New York: John Wiley, 1991. 900 p.

EGUCHI, E.S.; SILVA, E.L.; OLIVEIRA, M.S. Variabilidade espacial da textura e da densidade de partículas em um solo aluvial no Município de Lavras, MG. Revista Brasileira de Engenharia Agrícola e Ambiental, Campina Grande, v.6, n.2, p.242-6, 2002.

EMBRAPA. Manual de métodos de análise de solo. 2.ed. Rio de Janeiro: Ministério da Agricultura e do Abastecimento, 1997. 212 p.

EMBRAPA. Centro Nacional de Pesquisa de Solos. Sistema Brasileiro de Classificação de Solos. Rio de Janeiro, 1999. p.412.

FRAISSE, C.W.; SUDDUTH, K.A.; KITCHEN, N.R.; FRIDGEN, J.J. Use of unsupervised clustering algorithms of delineating within-field management zones. St. Joseph: American Society of Agricultural Enggineers, 1999. $121 \mathrm{p}$.

FRIDGES, J.J.; FRAISSE, C.W.; KITCHEN, N.R.; SUDDUTH, K.A. Delineation and analysis of sitespecific management zones. In: INTERNATIONAL CONFERENCE ON PRECISION AGRICULTURE, 5., 2000, Minneapolis. Proceedings... Minneapolis: ASA/CSSA/SSSA, 2000. 16 p. 
GONÇALVES, A.C.A.; FOLEGATTI, M.V.; MATA, J.D.V. Análises exploratória e geoestatística da variabilidade de propriedades físicas de um argissolo vermelho. Acta Scientiarum, Maringá, v.23, n.5, p.1149-57, 2001.

GONÇALVES, A.C.A.; FOLEGATTI, M.V. Correlação espacial entre retenção de água e textura do solo, para fins de manejo de irrigação. Engenharia Agrícola, Jaboticabal, v.22, n.3, p.296-303, 2002.

HALL, G.F.; OLSON, C.G. Predicting variability of soils from landscape models. In: MAUSBACH, M.J.; WILDING, L.P. Spatial variabilities of soils and landforms. Madison: Soil Science Society of America, 1991. p.9-25. (SSSA Special Publication, 28)

JOHNSON, G.A.; MORTENSEN, D.A.; GOTWAY, C.A. Spatial and temporal analysis of weed populations using geoestatistics. Weed Science, Champaign, v.44, n.3, p.704-10, 1996.

MACHADO, R.V. Variabilidade espacial de atributos físico-hídricos em uma hidrosseqüência de solos bem a muito mal drenados. 1994. 88 f. Dissertação (Mestrado em Solos e Nutrição de Plantas) Escola Superior de Agricultura de Lavras, Lavras, 1994.

MARQUES JÚNIOR, J.; LEPSCH, I.F. Depósitos superficiais neocenozóico, superfícies geomórficas e solos em Monte Alto, SP. Geociências, São Paulo, v.19, n.2, p.265-81, 2000.

McBRATNEY, A.B.; WEBSTER, R. Choosing functions for semi-variograms of soil properties and fitting them to sampling estimates. Journal Soil Science, Oxford, v.37, n.3, p.617-39, 1986.

POCAY, V.G. Relações entre pedoforma e variabilidade espacial de atributos de latossolos sob cultivo intensivo de cana-de-açúcar. 2000. 177 f. Dissertação (Mestrado em Ciência do Solo) Faculdade de Ciências Agrárias e Veterinárias, Universidade Estadual de São Paulo, Jaboticabal, 2000.

ROBERTSON, G.P. $G S^{+}$: Geostatistics for the environmental sciences - GS ${ }^{+}$User's Guide. Plainwell: Gamma Design Soffware, 1998. 152 p.

SAS, Statistical Analysis System for Windows. Computer program manual. Cary, 1995. 705 p.

SILVA, A.P.; LIBARDI, P.L.; VIEIRA, S.R. Variabilidade da resistência à penetração de um Latossolo Vermelho-Escuro ao longo de uma transeção. Revista Brasileira de Ciência do Solo, Campinas, v.13, n.1, p.1-5, 1989.

SOUZA, L.S.; COGO, N.P.; VIEIRA, S.R. Variabilidade de propriedades físicas e químicas do solo em um pomar cítrico. Revista Brasileira de Ciência do Solo, Viçosa, v.21, n.3, p.367-72, 1997.

SOUZA, C.K. Relação solo-paisagem-erosão e variabilidade espacial de latossolos em área sob cultivo de cana-de-açúcar no município de Jaboticabal (SP). 2001. 186 f. Dissertação (Mestrado em Ciência do Solo) - Faculdade de Ciências Agrárias e Veterinárias de Jaboticabal, Universidade Estadual Paulista, Jaboticabal, 2001.

SOUZA, Z.M.; BARBIERI, D.M.; VERTUAN, H.V.; MARQUES JÚNIOR, J.; PEREIRA, G.T.; BENTO, M.J.C. Variabilidade espacial de atributos físicos em um latossolo vermelho eutroférrico sob cultivo de cana-de-açúcar na região de Jaboticabal (SP). In: REUNIÃO BRASILEIRA DE MANEJO E CONSERVAÇÃO DO SOLO E DA ÁGUA, 14., 2002, Cuiabá. Anais... Cuiabá: Sociedade Brasileira de Ciência do Solo. 2002. 1 CD ROM.

SOUZA, C.K.; MARQUES JÚNIOR, J.; MARTINS FILHO, M.V.; PEREIRA, G.T. Influência do relevo e erosão na variabilidade espacial de um latossolo em Jaboticabal (SP). Revista Brasileira de Ciência do Solo, Viçosa, v.27, n.6, p.1067-74, 2003.

SURFER. Surfer 7.0. Contouring and 3D surface mapping for scientist's engineers. User's Guide. New York: Golden Software, 1999. 619 p. 
VIEIRA, S.R.; HATFIELD, J.L.; NIELSEN, D.R.; BIGGAR, J.W. Geoestatiscal theory and application to variability of some agronomical properties. Hilgardia, Oakland, v.51, n.1, p.1-75, 1983. VIEIRA, S.R. Variabilidade espacial de argila, silte e atributos químicos em uma parcela experimental de um Latossolo Roxo de Campinas (SP). Bragantia, Campinas, v.56, n.1, p.181-90, 1997.

WARRICK, A.W.; NIELSEN, D.R. Spatial variability of soil physical properties in the field. In: HILLEL, D. (Ed.). Applications of soil physics. New York: Academic Press, 1980. p.319-44.

WEBSTER, R. Quantitative spatial analysis of soil in the field. Advances Soil Science, New York, v.3, n.1, p.1-70, 1985. 